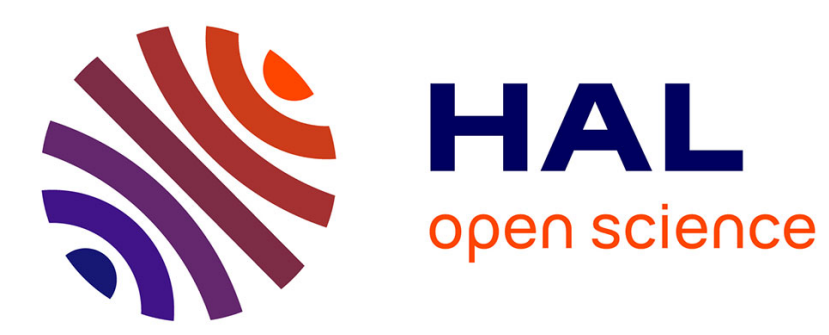

\title{
Le statut de la rente chez Sraffa, contribution à un débat récent
}

Lahouari Addi

\section{To cite this version:}

Lahouari Addi. Le statut de la rente chez Sraffa, contribution à un débat récent. Revue Economique, 1985, 18 p. halshs-00397779

\section{HAL Id: halshs-00397779 \\ https://shs.hal.science/halshs-00397779}

Submitted on 23 Jun 2009

HAL is a multi-disciplinary open access archive for the deposit and dissemination of scientific research documents, whether they are published or not. The documents may come from teaching and research institutions in France or abroad, or from public or private research centers.
L'archive ouverte pluridisciplinaire $\mathbf{H A L}$, est destinée au dépôt et à la diffusion de documents scientifiques de niveau recherche, publiés ou non, émanant des établissements d'enseignement et de recherche français ou étrangers, des laboratoires publics ou privés. 
Le statut de la rente chez Sraffa, contribution à un débat récent

Lahouari ADDI

Maître-assitant à l'Institut des Sciences sociales, Université d'Oran

In Revue Economique, Paris, №3, Mai 1985

Sommaire

- La rente comme ponction de la demande effective

- La formalisation du chapitre XI et sa rectification

- Ordre de fertilité des terres et ordre de rentabilité

Texte intégral

Je tiens à remercier les référées anonymes de la Revue économique pour leurs critiques sur une version antérieure de cet article : mais il va de soi que je demeure seul responsable de toute erreur y subsistant selon la formule désormais consacrée.

Les récentes controverses sur la rente, suscitées par le chapitre XI du livre de P. Sraffa [1], ont abouti à la conclusion selon laquelle l'ordre de fertilité des terres n'est pas celui de leurs rentabilités respectives et que cet ordre est fonction du système de prix. Il faut admettre que la réalité observable contredit ce résultat, mais l'important n'est pas tant la confrontation de la réalité observable au résultat énoncé que de comprendre comment les auteurs qui l'ont formulé, notamment C. Montani [2] et à sa suite B. Schefold [3], P. Vîdonne [4], etc., en sont arrivés à le formuler. La raison est que ces auteurs ont bâti leur démarche sur la formalisation mathématique du livre de Sraffa, formalisation reproduisant la conception de la rente comme coût de production et non comme ponction sur la demande effective. D'où l'autre conclusion à laquelle ont abouti les auteurs cités : la conceptualisation de Sraffa est en contradiction avec l'optique ricardienne de la rente.

Mais il faut souligner que ce chapitre XI, très court, n'est pas une fin en soi pour son auteur et qu'il introduit ou aide à comprendre la place du capital fixe, déjà amorti dans le système de production, assimilable au procès de production jointe ou à la terre qui procure une rente. Si le capital fixe amorti est encore en service, dit Sraffa, il faut l'assimiler à la terre qui rapporte une rente sans avoir entraîné un coût de production qui l'aurait justifié. Notre contribution à ce débat s'ordonnera autours de trois points :

a. Dans l'optique ricardienne, la rente n'est pus un coût de production. Elle est une ponction sur la demande effective, laquelle demande effective n'est que le produit net du cycle (ou des cycles) de production antérieur(s).

b. la formalisation mathématique du chapitre XI est erronée, du point de vue de la méthodologie d'ensemble du livre de Sraffa et de l'optique ricardienne de la rente, en ce qu'elle fait de la rente un coût de production. Cette formalisation appelle à une rectification dans le respect des hypothèses de Sraffa et sans conséquence sur la conceptualisation générale. 
c. Les controverses suscitées par ce fameux chapitre XI ont été toutes menées sur la base de cette formalisation mathématique erronée qui a mené vers l'inversion de l'ordre de fertilité des terres et celui des rentabilités respectives.

\section{La rente comme ponction de la demande effective}

Le mécanismes d'apparition et de financement de la rente est le même que ce soit au niveau de l'agriculture ou au niveau de l'industrie. Mais pour simplifier les choses, prenons l'exemple de la rente foncière et supposons trois terrains $\mathrm{A}$, $\mathrm{B}$ et $\mathrm{C}$, de qualité différente, mis successivement en culture pour répondre à la demande que ni la production du terrain $\mathrm{A}$, ni celle du terrain $\mathrm{B}$ n'ont pu satisfaire. D'où peut bien provenir la rente que perçoit le terrain $\mathrm{A}$

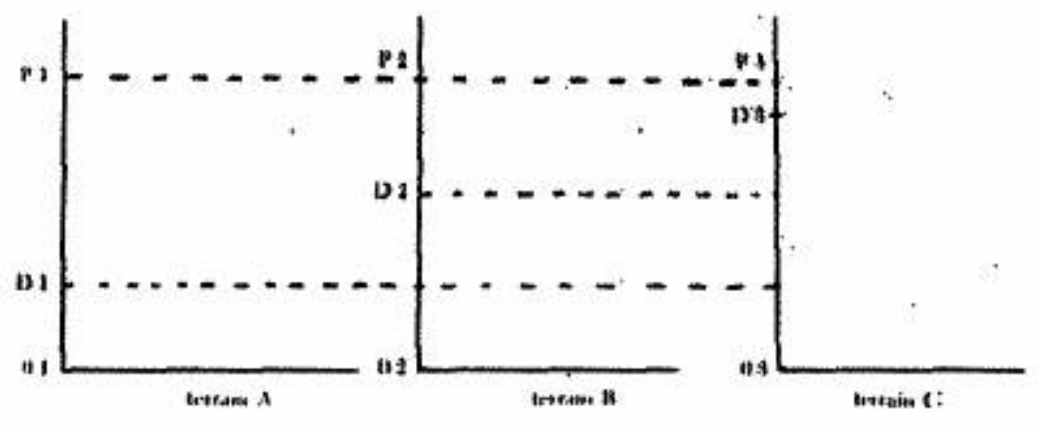

L'offre du terrain A fixe le prix de vente au seuil maximal qu'accepte la demande, prix qui lui procure une rente, en plus du taux de profit de la branche. C'est à la faveur de ce niveau du prix de vente, relativement élevé que les terrains $\mathrm{B}$ et $\mathrm{C}$ sont mis en culture. La demande accepte donc de payer $\mathrm{P} 1$ une marchandise dont les dépenses de production sont successivement D 1, D 2 et D 3. Pour que la rente n'existe pas, il faudrait que les prix soient de niveaux différents, c'est-à-dire $\mathrm{P} 1<\mathrm{P} 2<\mathrm{P} 3$. Or cette différence simultanée de prix d'un même produit est impensable en économie, en raison des tendances au nivellement que suscite la concurrence entre les acheteurs [5]. Pourquoi voudrait-on que l'offre du terrain A, et ensuite celle du terrain $\mathrm{B}$, fixent leurs prix àdes niveaux inférieurs à ceux pour lesquels la demande peut réaliser plus que le volume total de leurs productions ? La demande, dans ce cas, est dessaisie d'une fraction de la valeur et cette fraction n'est autre que la rente qui se forme dans la répartition et non dans la production. Le deuxième terrain dessaisit la demande d'une moindre fraction que le premier et le troisième ne prend rien qui ne soit justifié économiquement. Nous pouvons donc dire que la rente provient de la demande, qui accepte de payer $\mathrm{P} 1$ une marchandise pour laquelle les dépenses de production ont été respectivement $\mathrm{D} 1$ et $\mathrm{D} 2$. Ce sont des lois propres à la demande qui expliquent la formation de la rente.

Le mode de financement de la rente est le manque à gagner de la demande qui est ainsi dessaisie d'une fraction de la valeur sans aucune contrepartie économique. Mais ce dessaisissement intervient quand le produit net opère sa mutation en demande effective (salaires se dissolvant dans la consommation et profit dans l'investissement). La rente est ainsi financée non pas par les salaires et le profit de la branche où elle apparaît, mais par l'ensemble, des salaires et l'ensemble du profit qui s'adressent à la branche directement ou indirectement. Sa formation n'intervient qu'à la fin de la période du processus de production t0 et c'est à cause de la rente que le prix est une donnée exogène à la production, A la fin de la 
période t0, le produit net contient l'ensemble de la valeur créée, en dehors duquel il n'y a aucune autre valeur nouvelle.

La valeur sociale est irréductible au produit net, résultat d'un processus de création qui s'est étalé sur une période t 0 . Au cours de la période ultérieure $\mathrm{t} 1$, ce produit net va constituer la demande effective (consommation finale + investissement) d'où se détachera une fraction pour financer la rente et l'ensemble du travail improductif. Les rentes, comme le travail improductif, ne créant pas de valeur, en prennent au produit net par le biais de la demande effective. Ces ponctions empruntent un mécanisme rendu complexe par le double passage du produit net à la demande effective et de la période t0 à la période $\mathrm{t} 1$, complexité qui rend improbable la vérification comptable de l'équilibre entre la somme des manques à gagner et celle des rentes, car tous les flux susceptibles de véhiculer des rentes ne peuvent être connus et maîtrisés. C'est donc par hypothèse qu'il faudra poser l'égalité des rentes et des manques à gagner de la demande effective, au-delà de plusieurs successions de périodes, en attendant que la science économique se forge des instruments capables de vérifier mathématiquement cette hypothèse, Ce qui n'empêche pas d'apprécier les liens de causalité entre, d'une part, les salaires et le profit et, d'autre part, la rente, une fois le mécanisme de son apparition identifié.

Il faut comprendre que la rente n'apparaît pas selon un mécanisme de transfert de valeur du terrain marginal $\mathrm{C}$ vers le terrain $\mathrm{A}$. Comment est-il possible que le terrain marginal, qui arrive à peine à dégager un produit net composé des salaires et du profit, puisse dégager une valeur supplémentaire et qui, précisément, profiterait à un terrain plus fertile et logiquement créateur de plus de valeur.? [6]. On arriverait à soutenir qu'un terrain crée moins de valeur qu'un autre terrain moins fertile ! Du reste, les auteurs classiques n'ont jamais soutenu que la rente provenait du terrain marginal.

Lorsque la terre de troisième qualité est mise en culture; écrit Ricardo, une rente surgit immédiatement sur la terre de deuxième qualité; comme dans le cas précédent, elle est réglée par la différence entre leurs pouvoirs producteurs. En même temps s'accroît la rente sur la terre de première qualité, rente qui est toujours nécessairement supérieure à la rente sur la terre de seconde qualité en raison de la différence entre les produits que l'on peut obtenir sur l'une et sur l'autre à l'aide d'une quantité donnée de capital et de travail [7].

Quelle peut être la signification économique du manque à gagner de la demande? Peut-on assimiler la notion de manque à gagner à celle de rente négative? Le manque à gagner est l'expression qui désigne approximativement la ponction qu'opère la rente au détriment de la demande. Ce qui signifie qu'il y a transfert de valeur sans contrepartie, détournement d'une fraction de la valeur au détriment de la demande, valeur qui aurait pu être échangée contre une autre fraction équivalente de valeur [8]. La demande perd en quelque sorte dans l'échange sans que les sujets de la demande aient conscience de cette perte. On objectera que le terrain le plus fertile crée plus de valeur et qu'il n'y aurait donc pas de perte. Oui, mais au même prix, le terrain marginal crée moins de valeur.

Deux raisonnements sont alors possibles avec le même résultat pour la demande. Ou bien la demande finance la rente du terrain fertile ou elle finance le terrain marginal de telle manière à lui assurer le taux de profit moyen de la branche. Pour la demande, le résultat logique est le même ; elle se dessaisit d'une fraction de la valeur au profit de l'un ou de l'autre terrain. Pour les deux terrains, le résultat n'est pas le même, puisque l'un empoche la rente l'autre non ; du point de vue de l'économie globale, avec ou sans rente, le volume de valeur totale est identique, la rente ne créant aucune contrepartie. 
Mais si la rente n'est pas un coût de production, d'où provient cette insistance qu'ont de nombreux auteurs à la considérer comme tel? Il y a comme un malentendu, sur ce point, qu'il faut dissiper. La rente n'est pas un coût de production dans le cycle t0 où elle s'est manifestée en tant que telle. Si elle est financée par une fraction de la demande effective destinée à la consommation productive, elle devient un prix d'offre, un coût de production dans le cycle t1 et, dans cette perspective, la rente entre dans les coûts de production de ce cycle. Dire que la rente n'est pas un coût de production, cela signifie qu'elle n'est pas un coût de production de son propre cycle de production. Du fait que la réalité économique est dominée par la concurrence imparfaite et par la rareté relative des biens, le prix d'offre de n'importe quel bien contient un volume relativement élevé de rente lié au déséquilibre entre l'offre et la demande. La rente qu'attire le produit net du cycle t0 « se dissout» dans les coûts de production du demandeur au cycle t1. Ce demandeur n'a aucune conscience d'avoir payé une rente, sinon des coûts de production élevés ou moins élevés, et son objectif n'est pas tant de répercuter la rente qu'il a payée, sur l'acheteur, que de couvrir ses coûts de production et d'empocher autant que possible une rente, en plus du profit de la branche, en modulant le volume de sa production. Et ainsi de suite pour l'ensemble des agents.

Mais même considérée comme coût de production, un cycle t1, la rente n'entre pas directement en conflit avec le profit et les salaires de la branche. C'est-à-dire que le procès de sa formation n'amoindrit ni le profit, ni les salaires du terrain marginal, et ce pour deux raisons :

a. Les salaires que versent les fermiers aux ouvriers agricoles ont, à peu près, le même niveau. Tant qu'il y a des ouvriers agricoles qui acceptent des salaires plus bas que ceux versés, les fermiers nivellent par le bas les salaires. La terre marginale ne paie pas des salaires plus bas que ceux que verse la terre fertile, sinon celle-ci licencierait ses travailleurs pour recruter d'autres qui accepteraient le même niveau de salaire que celui de la terre marginale.

b. Le capital qui s'investit dans le terrain marginal obéit lui aussi à la concurrence des capitaux. S'il ne rapporte pas un taux de profit moyen dans la branche, il émigre ailleurs. II est prêt à renoncer à la rente mais non au profit.

Les terrains les plus fertiles n'ont pas des taux de profit supérieurs à ceux des autres terrains, mais ont des taux de rentabilité supérieurs. La différence entre les différents taux de rentabilité constitue précisément le taux de rente.

Cest pourquoi salaire et profit, dans le système étalon de Sraffa, peuvent fluctuer en raison inverse entre 0 et 1 dans le produit net étalon sans que la rente n'en soit affectée ou qu'elle ne les affecte. Elle entre en conflit avec le produit net de l'ensemble du système seulement lorsque le produit net se transforme en demande effective à une période ultérieure. C'est pour n'avoir pas perçu cette particularité dans la répartition que de nombreux auteurs, notamment C. Montani, abusés, il est vrai, par la formalisation erronée du chapitre XI, ont abouti à un résultat théorique discutable qui a consisté, d'une part, à faire télescoper le profit, les salaires et la rente sur un même vecteur-prix appartenant à la même période et, d'autre part, à inverser l'ordre de fertilité des terres et celui de leurs rentabilités respectives.

\section{La formalisation du chapitre XI et sa rectification}


Pour comprendre le contenu du chapitre XI, où il est question de rente foncière, il est indispensable de rappeler brièvement les éléments essentiels qui sous-tendent la méthodologie de Sraffa :

a. Des marchandises sont produites par d'autres marchandises dans un système d'autoreproduction (in a self replacing state) qui réunit plusieurs branches.

b. Le système ne laisse aucune place à la consommation finale : toute la production est destinée à la production.

c. Le système contient des marchandises fondamentales et des marchandises non fondamentales. Les premières entrent dans la production de toutes les marchandises, les secondes n'entrent que dans leur propre production.

d. Un système étalon peut être construit sur la base des seules marchandises fondamentales dans le respect des proportions de marchandises utilisées pour la production des autres marchandises.

La finalité de la construction de Sraffa est de déterminer les prix et le taux de profit, en tentant de montrer que les marchandises dites non fondamentales n'ont aucune incidence sur ce dernier [9]. Dès les premières pages de l'ouvrage, après avoir exposé les mécanismes de reproduction du système et après avoir distingué les marchandises fondamentales des marchandises non fondamentales, Sraffa précise le statut de ces dernières dans le système général :

... il y a place pour une nouvelle classe de produits, de « luxe» qui ne sont utilisés ni comme instruments de production, ni comme moyens de subsistance, dans la production des autres ((1970), p.9).

Le texte de Sraffa est très abstrait et se réfère très peu à la réalité concrète, mais à chaque fois qu'il donne des exemples de marchandises non fondamentales tirées de la réalité concrète, il cite les chevaux de course, les autruches, les oeufs d'autruche, la soie grège, ou des matières premières, des plantes de luxe, ou encore des fèves, Sraffa ne dit pas que les marchandises non fondamentales sont exclusivement des ressources naturelles, mais il précise au chapitre XI que les ressources rares qui procurent une rente peuvent être considérées comme marchandises non fondamentales. Il écrit :

Les ressources naturelles qui sont utilisées dans la production comme la terre et les gisements minéraux et qui, étant rares, permettent à leurs propriétaires d'obtenir une rente, peuvent être considérées comme ayant, parmi les moyens de production, une position équivalente à celle des biens non fondamentaux parmi les produits ((1970). p. 91).

D'où la position de Sraffa à considérer la terre, qui rapporte une rente à son propriétaire, comme une marchandise non fondamentale.

La finalité n'est pas tant d'exclure du système étalon la terre ou autres matières premières (le chapitre XI consacré à la terre ne s'étale pas sur plus de six pages) qui procurent une rente que d'intégrer dans la problématique le capital fixe amorti qui procure une rente et qui est, en conséquence, exclu du système étalon. 
Le système d'équations représentant la production d'une marchandise «g» est le suivant, traçant l'évolution de l'amortissement de la machine $\mathrm{M}$ :

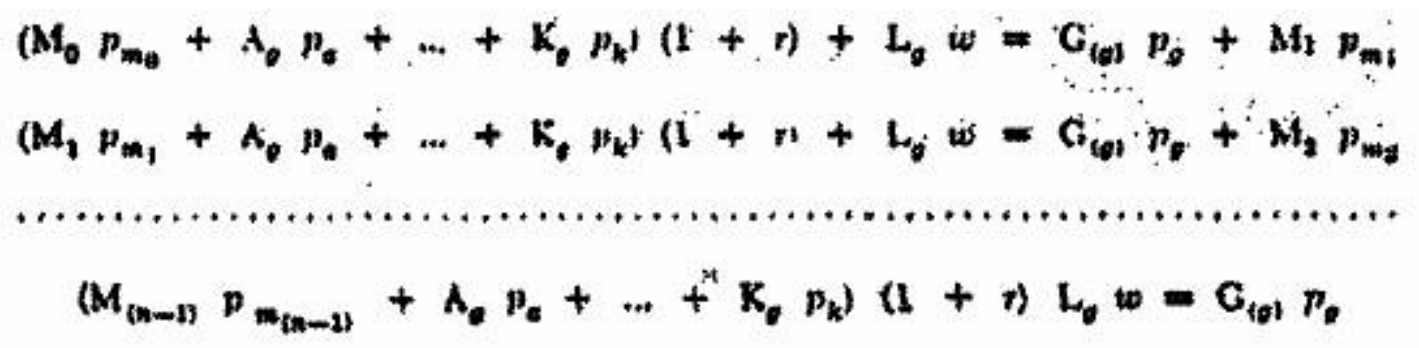

Que devient ce système quand la machine $M_{n} p_{M n}$ est amortie et représente un coût théoriquement nul? Puisqu'elle demeure en fonction et qu'elle procure une "rente » en quelque sorte, que devient le système avec une machine qui fonctionne, par exemple, trois années après son amortissement et dont le symbole serait $\mathrm{M}_{(\mathrm{n}+3)} \mathrm{p}_{\mathrm{M}(\mathrm{n}+3)}$ ? Deux questions vont alors se poser : quelle est la place de $\mathrm{M}_{(\mathrm{n}+3)} \mathrm{p}_{\mathrm{M(n+3)}}$, dans le système d'équations de production de la marchandise $g$, sachant que son coût de production est nul ? D'où provient la rente que procure la machine en question déjà amortie, sachant qu'elle ne fera pas partie du système étalon?

Dans le respect de la méthodologie d'ensemble de Sraffa, le capital fixe amorti disparaît des coûts de production. Aussi, n'est-il pas possible de continuer d'écrire les équations précédentes avec $M_{n} p_{M n} M_{n+1} p_{M n+1 \ldots}$ du côté gauche jusqu'à élimination de la machine du procès de production. La valeur $\mathrm{M}_{n} \mathrm{p}_{\mathrm{Mn}}$ est nulle et sa place dans les coûts de production ne se justifie plus. La difficulté est qu'elle procure une valeur dont l'origine demeure inexplicable, il est exclu que cette valeur provienne du profit ou des salaires de la branche car il n'y a aucune raison de laisser en fonction une machine qui grèverait le profit et les salaires. D'un autre côté, le produit de la branche, c'est-à-dire le côté droit de l'équation, se trouve augmenté de la valeur que procure la machine amortie, valeur exprimée dans la marchandise produite. Aussi, bien que $\mathrm{M}_{n} \mathrm{p}_{\mathrm{Mn}}$ soit nul en tant que coût de production, il y a toujours un coefficient $\rho$ tel que la valeur que rapporte $M_{n} p_{M n}$ soit égale à

\section{$P_{\phi} \mathrm{G}_{(0)} P_{0}$}

$\rho_{0} \rho_{1} \rho_{2} \ldots$ étant les coefficients de la $1^{\mathrm{er}}$ année, $2 \mathrm{e}$ année, $3 \mathrm{e}$ année ... après l'amortissement de la machine.

Dès lors, $\rho_{0} \mathrm{C}_{(\mathrm{g})} \rho_{1} \mathrm{C}_{(\mathrm{g})} \rho_{2} \mathrm{C}_{(\mathrm{g})} \ldots$ sont des quantités de valeur qui s'ajoutent au produit de la branche selon l'équation :

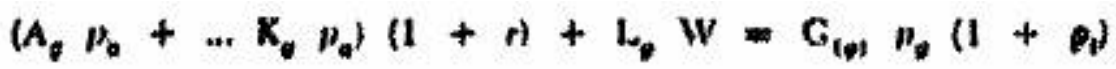

$i$ étant la ième année après la fin de l'amortissement de la machine. Dans la formalisation du paragraphe 76, la valeur de la machine non amortie est du côté droit en tant que produit joint qui s'ajoute à l'output de la branche. Mais sitôt amortie, la machine disparaît du système d'équations, bien qu'elle soit encore en service et procure de la valeur. Aussi, théoriquement, n'est-il pas légitime de faire apparaître du côté droit la valeur qu'elle procure puisqu'elle n'est pas un coût de production? La demande des marchandises, dans un système qui s'autoreproduit, est simultanément leur offre et la valeur de l'output d'une branche se répartit dans 
les inputs des autres branches. Dès lors, les marchandises produites par des branches qui utilisent en partie du capital fixe amorti contiennent une quantité de la valeur assimilable à la rente. La valeur que procure le capital fixe amorti, ne provenant ni des salaires, ni des profits, ne peut provenir que de l'échange marchand, donc de la demande effective. Pour cette raison, le capital fixe amorti est exclu du système étalon ; il ne crée aucune valeur, il en prend à la demande effective. La valeur que procure le capital fixe amorti, étant contenue dans la valeur de l'output de la branche qu'elle augmente d'autant, devrait logiquement figurer au côté droit des équations du système.

Ceci implique que la rente foncière comme toute autre rente, du fait de son caractère de ponction de la demande effective, devrait se trouver du côté droit des équations. Aussi, la formalisation mathématique du chapitre XI de l'ouvrage de Sraffa contient une ambiguïté qu'il faut lever dans le respect de la méthodologie d'ensemble.

La formalisation du chapitre XI est ainsi présentée :

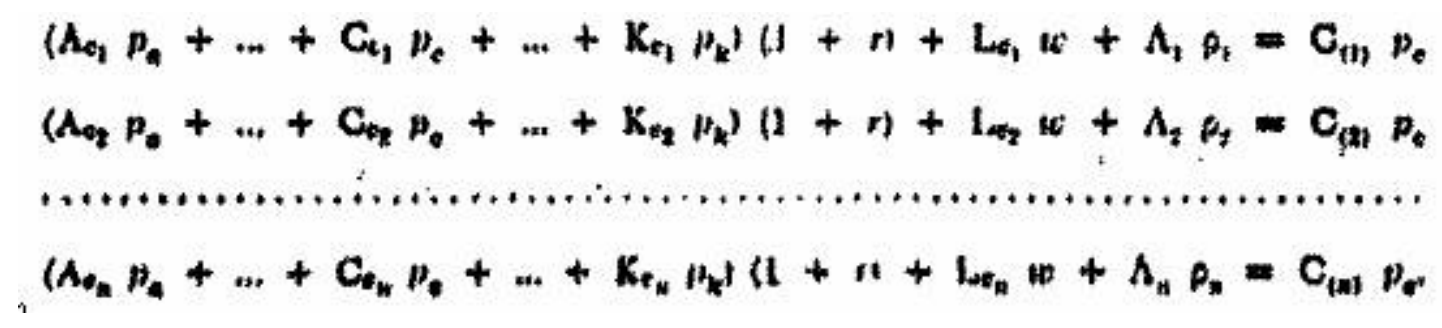

Cette formalisation suscite trois remarques :

a. Sraffa souligne que les rentes «n'apparaissent que d'un côté seulement du procès de production $\gg$ (p. 91) et c'est la raison pour laquelle il est impossible de les intégrer dans le système étalon, considérées ainsi comme des marchandises fondamentales. Si elles ne doivent apparaître que d'un côté du procès de production, il faudra alors justifier lequel : n'étant pas des coûts de production, elles ne peuvent apparaître que dans le côté droit des équations. Pourquoi alors le terme $A_{n} \rho_{n}$ affecté d'un signe positif, se trouve-t-il du côté gauche de l'équation alors qu'il n'est pas un coût de production ? Pourquoi n'est-il pas exprimé en valeur ?

b. Par ailleurs, une formalisation de ce type suppose non seulement que la rente est un coût de production, mais elle suppose aussi que le système général n'est composé que d'équations de production agricole (la marchandise $\mathrm{C}$ représentant le blé). Or limiter le système économique aux différentes terres et aux différentes techniques de production agricole, c'est l'amputer des parties où, précisément, la rente trouve l'origine de son financement, outre que du système atrophié en question, il est difficile d'extraire le système étalon. La formalisation du chapitre XI restreint l'économie à l'agriculture ce qui suppose que la rente trouve sa source de financement dans l'agriculture et que les prix de produits agricoles sont endogènes.

c. La dernière remarque enfin est relative à l'une des hypothèses de l'approche de Sraffa qui interdit de concevoir le système d'équations dans une perspective dynamique où les cycles de production se succéderaient. Le système s'auto-reproduit en un seul cycle de production, en se créant et se recréant à chaque cycle, les marchandises produites servant à produire d'autres marchandises. Autrement dit, la mutation du produit net en demande effective, chez Sraffa, est simultanée, ce qui ne permet pas d'entrevoir les flux en valeur qui existent d'un cycle de production à un autre. Mais pour ne pas compliquer cette formalisation (ce qui est possible en 
imaginant le même système utilisant des inputs de $t_{n}$ et produisant les outputs de $t_{n+1}$ ), nous continuerons à respecter l'hypothèse de " self replacing state » en acceptant la simultanéité du produit net et de la demande effective.

C'est pourquoi, nous semble-t-il, que la formalisation du chapitre XI doit être rectifiée et ce, dans le respect des hypothèses de Sraffa. Il faudra qu'elle restitue l'ensemble des branches de la reproduction sociale et qu'elle ne suppose pas que la rente soit un coût de production. Pour cela, elle devrait être de la forme :

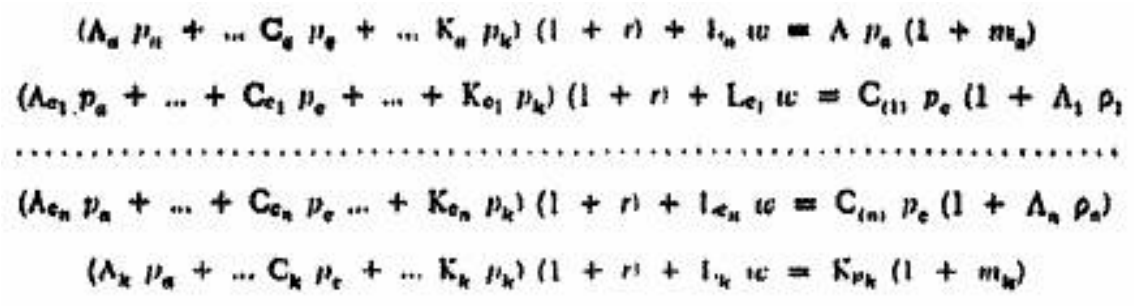

La rente foncière se réalise dans le système global comme si des fractions de valeur de $\mathrm{A}$..., de $K$, etc.. s'échangeaient contre des quantités $A_{1} \rho_{1} C_{(i)} p_{c}$. Ce coefficient $\rho$ i qui augmente d'une quantité de valeur la branche $\mathrm{C}$ doit avoir son vis-à-vis dans les branches $\mathrm{A}$... K, qui est un coefficient qui diminue d'autant $\mathrm{A}$... K tel que

$$
m_{k}+A p_{a}+\ldots m_{k} \cdot \mathrm{K} p_{k}+A_{i} p_{t} \cdot \mathrm{C}_{1 \xi} p_{c}=0
$$

Ma...Mk seraient les coefficients de ponction des branches A ... K qui déterminent le volume de valeur qui finance la rente dans la branche $\mathrm{C}$. Le coefficient de ponction, nombre négatif, apparaît dans les branches utilisatrices de marchandises qui procurent une rente. Supposons que les $\mathrm{A}$... $\mathrm{K}$ utilisent l'output de la branche $\mathrm{C}$ dans leurs procès de production respectifs. Supposons Ai, la surface de la terre i paramètre connu. La rente que procure la marchandise C(i) sera de volume : C(i) Ai $\rho_{\text {i. }}$ Quelles peuvent être les limites maximale et minimale de $\rho i$ ?

L'une des conditions du système est que :

$$
m_{a} \cdot A p_{k}+\ldots m_{k} \cdot K p_{0}+\ldots p_{1} C_{i n} p_{c}+\ldots C_{i n} n_{c}=n
$$

Ma ne peut pas annuler l'output de la branche A, et encore moins le rendre négatif, puisque les salaires et le profit de A étant positifs, l'output sera forcement positif. Mais Ma. étant un coefficient de ponction, il est par hypothèse inférieur à zéro. Aussi, Ma varierait entre 0 et -1.

D'où $0>\mathrm{M}_{0}>-1$.

Une autre démarche plus globale peut être aussi envisagée. Soit N l'ensemble des branches non agricoles qui réalisent la production des branches agricoles.

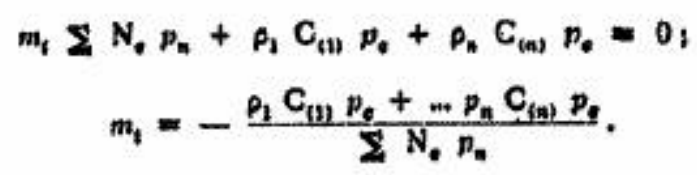


$\Sigma$ Nc $p_{n}$ étant positif, les coefficients de rente $\rho_{1, \ldots} \rho_{n}$ étant positifs par définition, $M_{1}$ ne peut être que négatif. D'un autre côté, $\mathrm{M}_{1}$ ne peut pas être inférieur à -1 ,. sinon la ponction en valeur serait supérieure à la valeur d'où elle est prélevée. D'où $0>\mathrm{M}_{1}>-1$..

$\rho_{1 \ldots} \rho_{\mathrm{n}}$ se partageront la valeur que déterminera le coefficient global $\mathrm{M}_{1}$ en fonction des surfaces $A_{1} \ldots A_{n}$. La limite maximale de $\rho_{1+\ldots} \rho_{n}$ est telle que le niveau des rentes ne peut pas être supérieur aux valeurs ponctionnées.

Une firme en situation de monopole empoche une rente dite de monopole, financée par la demande effective à laquelle s'adresse cette firme. La somme de cette rente avec la ponction en question est nulle, d'où la pertinence de la méthodologie de la marchandise non fondamentale qui n'a aucune incidence ni sur le produit net, ni sur les salaires et les profits de la branche. Au niveau de l'output général de l'ensemble du système d'équations, les rentes et les ponctions disparaissent car une firme prélève une quantité de valeur déjà créée sur une autre, en modifiant la répartition sans modifier le volume global de la production, la rente étant une ponction et non une création ex nihilo de valeur. Sraffa l'assimile à juste titre à une marchandise non fondamentale qui n'affecte ni le produit net du système ni le système étalon. Cest pourquoi l'hypothèse de la concurrence, parfaite ou imparfaite, chez Sraffa, est inutile puisqu'il est arrivé à exclure la rente de monopole du système étalon.

Selon qu'il y ait un excédent ou un manque par rapport à la demande (appréciée sur la base de son élasticité) la valeur de la marchandise de la branche (assimilée provisoirement à une firme) augmente (ou diminue) d'un volume égal à la rente positive (ou négative). C'est dans ce sens, nous semble-t-il, que Sraffa parle de rente négative au chapitre XI. Le volume de la rente négative connaît un seuil minimal fixé par le coefficient Ma qui ne saurait être inférieur à -1 , sinon il annulerait l'output de la branche $\mathrm{A}$, ce qui est une impossibilité économique en soi. La limite maximale est théoriquement la valeur totale de l'output de l'ensemble de l'économie, mais cette limite maximale est théorique et n'est jamais atteinte en raison de la concurrence qui amoindrit la rente de monopole.

\section{Ordre de fertilité des terres et ordre de rentabilité}

La controverse sur la rente, suscitée par le chapitre XI de l'ouvrage de Sraffa, tourne autour de la relation entre le niveau de fertilité des terres et le niveau de leurs rentabilités respectives. A partir de la définition de la pensée classique de la rente et de la formalisation du chapitre XI, les auteurs qui ont pris part au débat, à la suite de G. Montani, ont débouché logiquement sur une relation inversée des niveaux de fertilité et de rentabilité des terres. En tant qu'aboutissement d'une construction théorique, le résultat est logique, mais il est démenti par la réalité. Quand deux terrains sont de qualité différente, il n'y a aucune base commune de comparaison de leurs niveaux de fertilité avec leurs taux de rentabilité, à moins qu'ils produisent le même produit, Si les produits sont identiques, le blé par exemple, il serait pour le moins paradoxal de soutenir que le terrain le plus fertile est le moins rentable. Si les produits ne sont pas identiques, le blé d'un côté, les pommes de terre de l'autre par exemple, il n'y a aucune relation logique entre niveaux de fertilité et taux de rentabilité puisque, en effet, le terrain le moins fertile peut être le plus rentable et vice versa. La fertilité est une qualité physico-chimique et la rentabilité une donnée économique : leur relation ne peut être établie qu'en cas de même produit pour les deux terres et cette relation est sans équivoque. Une terre 
quelconque peut être plus rentable qu'une autre terre, plus fertile, si la production de la première terre rapporte plus en valeur que celle de la seconde. Mais, là, la concurrence poussera la seconde terre à cultiver le produit de la première terre et à empocher une rente si l'élasticité de la demande de ce produit le permet. Cette mise en relation singulière des ordres de fertilité et de rentabilité aboutit à une situation pour le moins contradictoire dans laquelle la terre la plus fertile est la terre marginale puisqu'elle est la moins rentable !

G. Montani écrit le système d'équations suivant :

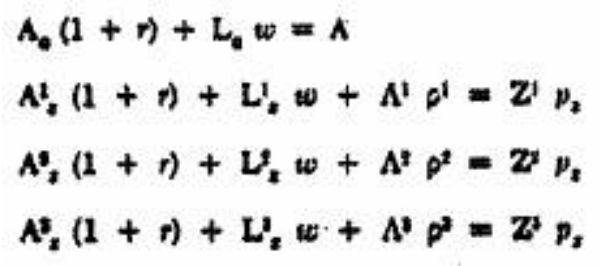

avec A la quantité de marchandises produite par la branche A : $\mathrm{A}^{\mathrm{H}} \mathrm{Z}$ la quantité de marchandises $\mathrm{A}$ utilisée par la terre $\mathrm{H}$ dans la production de $\mathrm{Z}$;

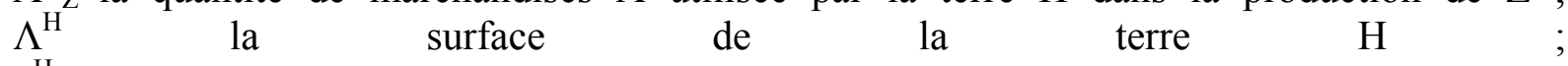
$\rho^{\mathrm{H}}$ le taux de rente de la terre $\mathrm{H}$.

Ce système d'équations peut représenter l'ensemble du système économique puisque, contrairement à celui du chapitre XI de l'ouvrage de Sraffa, il n'y a pas que des équations de production agricole. Mais il pose néanmoins problème en ce que la branche $\mathrm{A}$ ne consomme pas des produits des procès de $Z^{1}, Z^{2}$ et $Z^{3}$. Cette critique n'est pas formelle, car l'échange s'établit dans les deux sens, sans quoi il ne peut avoir lieu, et s'il n'y a pas échange, la rente ne pourra pas se réaliser. Il ne faut pas oublier que l'une des sources de Sraffa,. comme il le dit lui-même, est le tableau économique de F .Quesnay.

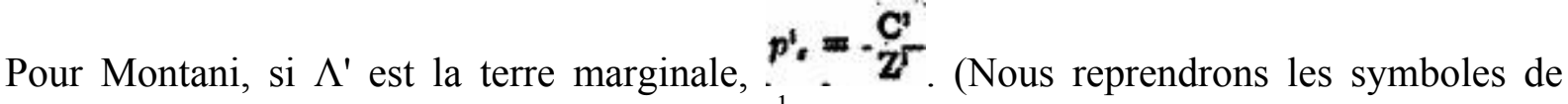
Montani pour simplifier les termes du débat) $\mathrm{p}_{\mathrm{z}}{ }_{\mathrm{z}}$ est le prix de la marchandise $\mathrm{z}$ produite sur la terre $\Lambda^{1} ; C^{1}$ serait son coût de production et $Z^{1}$ la quantité produite. Lorsque $\Lambda^{1}$ devient la terre marginale, le prix du blé deviendra $p^{*}=\frac{\mathbf{C}^{*}}{\mathbf{Z}^{\mathbf{z}}}$. S'il devient nécessaire de cultiver la terre $\Lambda^{3}$, le prix du blé devra augmenter jusqu'à atteindre $\boldsymbol{P}_{2}^{2}=\frac{\mathbf{C}^{2}}{\mathbf{Z}^{2}}$. A ce prix du blé, la rente sur le terrain $\Lambda^{1}$ s'accroîtra et il en surgira également une sur la terre $\Lambda^{2}$. Cependant, puisque au prix $\mathrm{p}^{3} \mathrm{z}$ du blé, la productivité par hectare de la terre $\Lambda 2$ est supérieure à celle de la terre $\Lambda^{1}$, le taux de rente sur la seconde terre sera supérieur à celui de la première $\left(\rho^{2}>\rho^{1}\right)$. L'ordre de fertilité et l'ordre de rentabilité ne coïncident plus » (Montani, op. cit., p. 137). L'erreur de C. Montani est qu'il déduit l'origine de la rente de l'augmentation des prix de $\mathrm{p}^{1} \mathrm{z}$ à $\mathrm{p} 2 \mathrm{z} . .$. alors que la mise en culture du terrain marginal intervient à la faveur du prix déjà en vigueur, c'està-dire celui de la première terre. 1.es prix $\mathrm{p}^{1} \mathrm{z} \mathrm{p}^{2} \mathrm{z} \mathrm{p}^{3} \mathrm{z} \ldots$ sont de même niveau et la rente surgit alors que les prix ne se modifient pas lors de la mise en culture de la terre nouvellement marginale. Au contraire, ils risquent de diminuer puisque l'offre globale augmente, sans que la proportion de cette diminution n'affecte le taux de profit. De ce point de vue, Montani ne respecte pas l'optique ricardienne de la rente. "Corn is not high because a rent is paid, but a rent is paid because corn is high" (Ricardo, Principles...). C'est de l'inversion de l'optique ricardicnne que Montani déduit que « contrairement à ce qu'affirme Ricardo, remarquons que l'ordre de fertilité des terres et l'ordre de rentabilité ne coincident pas » (Montani (1977), 
p.124). II faudra corriger en conséquence le raisonnement de G. Montani en tenant compte du fait que les prix ne se modifient pas les uns par rapport aux autres car la concurrence nivelle les prix des mêmes produits [10].

Si $\Lambda^{1}$ est la terre marginale, le prix du produit sera $\boldsymbol{p}^{1}=\frac{C^{n}}{\mathbf{Z}^{1}}$. Lorsque $\Lambda^{2}$ devient la terre marginale dans un premier temps et $\Lambda^{3}$ dans un deuxième temps, les prix, qu'ils se modifient ou non, seront d'un même niveau.

$$
p_{1}^{\prime}=p_{2}^{2}=p_{2}^{2}
$$

sachant que les coûts unitaires sur $\Lambda^{1} \Lambda^{2}$ et $\Lambda^{3}$ seront inégaux

$$
\begin{aligned}
& \mathrm{C}^{2}>\mathrm{C}^{2}>\mathrm{C}^{\prime} \\
& A^{3} p^{2}=0 \\
& A^{\prime} p^{\prime \prime}=\sigma^{\prime}-C^{\prime} \\
& A^{\prime} P^{\prime}=\left(C^{\prime}-C^{\prime}\right)+\left(C^{\prime}-C^{\prime}\right)=C^{\prime}-C^{\prime}
\end{aligned}
$$

L'égalité des prix découlera de l'égalité :

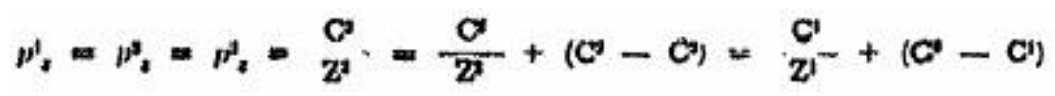

La rente apparait chez Montani comme un élément qui entre dans le coût de production de $\Lambda^{1}$ , d'où l'augmentation du coût unitaire, alors qu'en fait elle apparaît en comparant les coûts de production de terrain de qualité différente à l'avantage du plus fertile.

Mais cette critique n'est pas la seule. Le système d'équations reproduit l'erreur du chapitre XI et doit être, par conséquent, rectifié si l'on veut qu'il appréhende la rente à laquelle il donne naissance.

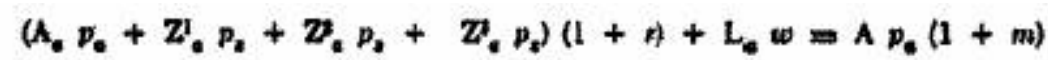

$$
\begin{aligned}
& \left(A_{2}^{\prime} p_{0}+Z_{1}^{1} p_{9}\right)\left(1+n+L_{1}^{\prime} N=Z^{1} p_{z}\left(1+A^{1} p^{\prime}\right)\right.
\end{aligned}
$$

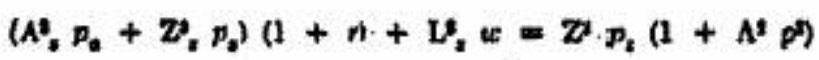

$$
\begin{aligned}
& \left(A_{2} p_{c}+D_{1} p_{2}\right)(1+\eta)+L_{1} w=Z_{p_{2}}\left(1+A^{\prime} p^{\prime}\right)
\end{aligned}
$$

avec comme hypothèses :

$\mathrm{A}^{1} \mathrm{z}$ pa $<\mathrm{A}^{2} \mathrm{z}$ pa $<\mathrm{A}^{3} \mathrm{zpa}$ par surface unitaire. $\mathrm{L}^{1} \mathrm{z}<\mathrm{L}_{\mathrm{z}}^{2}<\mathrm{L}^{3} \mathrm{z}$ par surface unitaire. Ces deux hypothèses se justifient par le fait que la terre marginale, du fait de sa moindre fertilité, consomme une plus grande quantité de capital et de travail par surface unitaire (ou produit unitaire) que la terre plus fertile. Les autres hypothèses fixent les limites maximale et minimale de $\mathrm{m}, \rho^{1}, \rho^{2}$ et $\rho^{3}$. Nous supposons que le paramètre $\Lambda^{\mathrm{H}}$ est connu.

$$
\begin{aligned}
& m \cdot A p_{q}+p^{\prime} Z^{\prime} p_{1}+p^{A} z^{\prime} p_{2}+p^{2} Z^{\prime} p_{1}=0_{i}
\end{aligned}
$$

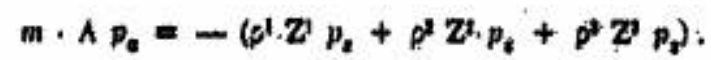


Par définition, la production de n'importe quelle branche est forcément positive et la rente sur la terre marginale est nulle. D'où $A p_{a}>0$ et $\rho^{3}=0$.

Cela implique que $\mathrm{m}$ doit être un nombre obligatoirement négatif pour écrire l'égalité :

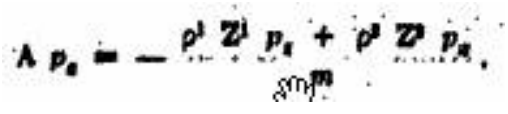

Comme $\operatorname{Ap}_{\mathrm{a}}(1+\mathrm{m})>0$, en tant que produit de la branche $\mathrm{A}, \mathrm{m}$ doit être supérieur à -1 .

D'où $0>m>-1$.

Si m est connu, $\rho^{1}$ et $\rho^{2}$ peuvent être déduits de l'égalité précédente et vice versa.

Dans le cas où il y aurait plusieurs branches $\mathrm{A}, \mathrm{B}$... K concernées par le financement de la rente, un taux global de financement pourrait être construit.

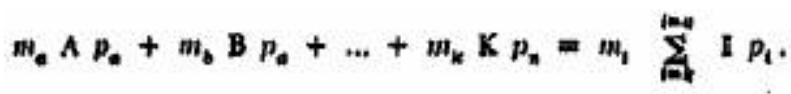

$m_{i} \sum_{i=0}^{i=0} I p_{i}$

serait la valeur que se partageront les coefficients de rente $\rho^{1}, \rho^{2}$ et $\rho^{3}$ relativement aux niveaux de fertilité et de rentabilité des terres $\Lambda^{1}, \Lambda^{2}, \Lambda^{3} \ldots \Lambda^{\mathrm{H}}$. Le coefficient $m_{1}$ ne devrait apparaître que dans les branches utilisatrices des produits qui procurent une rente telle que

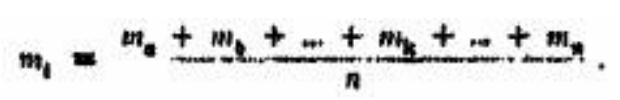

S'il y a $n$ branches concernées, $m_{1}$ serait le taux global de prélèvement ou de financement de la somme des rentes qui se forment dans le système. En cas d'abondance et d'homogénéité des terres, $m_{1}$ serait nul, ce qui entraînerait la nullité des rentes, $\rho^{1}, \rho^{2} \ldots \rho^{\mathrm{H}}[11]$.

Les auteurs qui, à la suite de Montani, ont commenté le chapitre XI, se sont appuyés sur la formalisation mathématique erronée de ce chapitre et ont conclu à une contradiction dans la conception de la rente chez Sraffa et chez Ricardo. Mais ils ont considéré la rente soit comme un coût de production, soit comme le troisième paramètre de la répartition, soit encore, les deux à la fois. A partir de cette confusion, ils ont cru pouvoir soutenir que le profit et le salaire pouvaient varier dans le même sens puisque la rente pouvait abaisser ou accroître et les profits et les salaires. Le télescopage des profits, des salaires et de la rente dans un même espacetemps d'une même branche conduit donc à des positions théoriques soit contraires à la rationalité économique, soit en contradiction avec la définition classique de la rente. Si l'on introduit la rente dans le produit net du système de Sraffa, alors que sa méthodologie l'interdit puisque la rente est assimilée à une marchandise non fondamentale; il ne peut y avoir que malentendu comme en témoigne cette phrase de P. Vidonne : «Or, nous savons quelle est la signification d'un étalon avec composantes négatives : la règle suivant laquelle la baisse du salaire exprimée dans un étalon quelconque entraîne une hausse du profit n'est maintenant plus vérifiée [12]. " Pourtant, la méthodologie du système étalon enrichit l'approche ricardienne de la rente en ce qu'elle délimite la source de la rente - le produit net du système 
étalon qui est aussi le produit net du système concret — en l'assimilant à une marchandise non fondamentale.

Mais P. Vidonne ne peut trouver l'origine de la rente avec la formalisation mathématique sur laquelle il réfléchit et qui est du type :

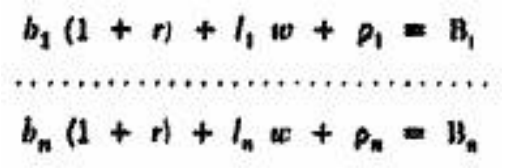

b1...bn étant la quantité de blé utilisée pour la production des quantités B1 ... Bn.

$\rho^{1} \ldots \rho^{\mathrm{n}}$ étant la rente des terres $1 \ldots \mathrm{n}$.

De là, on comprend le résultat contestable auquel aboutit $\mathrm{P}$. Vidonne pour qui la terre la moins fertile paye une rente différentielle. Il est légitime de se demander comment une terre relativement peu fertile, ayant des coûts de production unitaires élevés puisse payer en sus une rente, à moins d'en faire la terre la plus rentable. D'où aussi la conclusion selon laquelle « la logique du logique du modèle de rente avec terre homogène telle que la formule Sraffa conduit à une impasse » [13]

L'interprétation de B. Schefold [14] n'est pas acceptable, non plus, car cet auteur réfléchit sur une formalisation dont une des hypothèses est en contradiction avec l'objet de la question.

Le raisonnement de Schefold est le suivant :

soit k1 le coût du capital (matières premières et salaires) sur le premier terrain ou pour la première technique ;

soit $\mathrm{k} 2$ le coût du capital (matières premières et salaires) utilisé sur le second terrain moins fertile;

soit $\Lambda 1$ la surface nécessaire par unité de produit pour le premier terrain ou la première technique;

soit $\Lambda 2$ la surface nécessaire par unité de produit pour le premier terrain ou la première technique

Par hypothèse, B. Schefold écrit :

$$
\begin{aligned}
& k_{2}<k_{2} \\
& \Lambda_{1}>\Lambda_{2}
\end{aligned}
$$

La première hypothèse est logique du fait que les coûts de production unitaires sur le terrain marginal sont supérieurs à ceux du premier terrain. Par contre, la seconde hypothèse n'est pas acceptable : si le premier terrain est plus fertile ou la première technique est plus productive, logiquement la surface par unité produite dans le second terrain est supérieure à celle du premier terrain. Il est impossible d'inverser l'hypothèse sans contredire l'objet de la réflexion 
qui porte sur des terrains de qualité différente dont l'un est forcément plus rentable par unité produite [15].

Mais ne nous arrêtons pas là et voyons pourquoi B. Schefold pose cette hypothèse viciée. En partant de la conception de la rente comme coût de production, la formalisation de $\mathrm{B}$. Schefold ne donne une rente positive que si est posée comme hypothèse l'inégalité $\Lambda 1>\Lambda 2$. Cette hypothèse irréaliste est posée pour se dégager d'une perspective sans issue. Rappelons la démarche de B. Schefold.

dioù

$$
\begin{aligned}
& k_{1}+k A_{1}=p \\
& k_{2}+k A_{2}=p
\end{aligned}
$$

$$
=\frac{k_{1}-k_{1}}{\Lambda_{2}-\Lambda_{1}}
$$

La rente n'est positive qu'a la condition que $\Lambda 1>\Lambda 2$. Si on rétablissait cette hypothèse dans son sens réel, c'est-à-dire $\Lambda 1<\Lambda 2$, la rente serait négative, ce qui est une impossibilité puisque la rente nulle est cette du terrain marginal et les rentes positives celles des terrains plus fertiles,. La rente négative entraîne ipso facto l'élimination du terrain concerné de la compétition économique,

Si la démarche précédente avait respecté l'optique ricardienne de la rente qui veut que celle-ci soit une ponction et non un coût de production, elle n'aurait pas abouti à ce résultat singulier incompatible avec la réalité observable. Reprenons cette démarche, en la corrigeant, sans partir d'aucune hypothèse restrictive. Soit deux terrains $t 1$ et $t 2$ dont nous ne savons pas lequel est le plus grand et $\mathrm{C} 1$ et $\mathrm{C} 2$ les coûts de production dont nous ignorons lesquels sont les plus élevés.
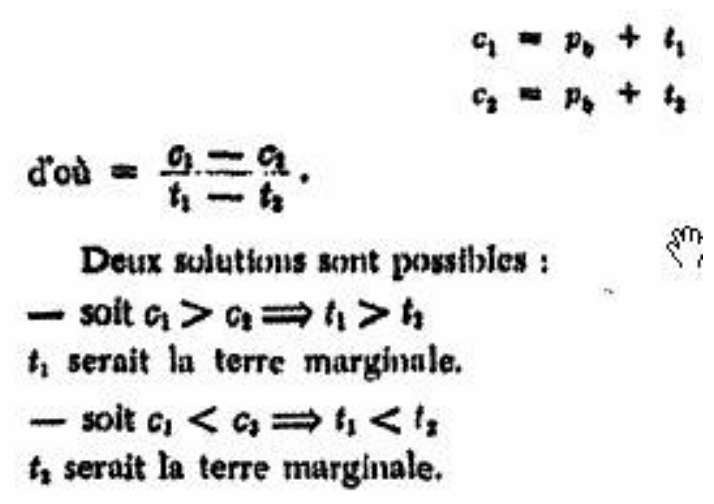

L'optique ricardiennc de la rente n'est donc pas en rupture avec la logique formelle ni avec la réalité observable.

Le mode de calcul ou d'identification est le même selon qu'il s'agit de la rente absolue ou de la rente différentielle. En cas de terre abondante et homogène, il n'y a ni rente absolue, ni rente différentielle. En cas de terre abondante de fertilité différente, la rente absolue est nulle mais la rente différentielle existe à la faveur de la différence des coûts unitaires de production. Au lieu que cette différence bénéficie à la demande, elle bénéficie au propriétaire du terrain plus fertile. Quand les terres sont rares et de qualité différente, la rente différentielle I, II, III... s'ajoute à la rente absolue, à travers le même mécanisme d'apparition, celui de la ponction sur la demande. 
En conclusion, le récent débat mené dans le cadre des commentaires du chapitre XI du livre de Sraffa a été marqué par un déplacement dans l'explication de la rente de la ponction sur la demande effective, qu'elle est, vers le coût de production dans la branche qu'elle ne saurait être. Ce déplacement a été à l'origine des confusions et des malentendus qui ont mené droit vers des conclusions irrecevables et incompatibles avec la réalité empirique et ledit postulat de la rationalité des agents économiques [16].

\section{ANNEXE}

Soit $\boldsymbol{p}_{b}$ ot $\boldsymbol{p}_{\mathrm{b}}$ les prix unitaires de production de $t_{1}$ et $t_{2}$ sans tenir comapte do la rente. Par hypothòse $\mu_{\mathrm{b}} \neq p_{\mathrm{b}}^{2}$. La rute intervient pour égaliser les prix en fin de procis, c'est-it-dire nu marché.

On peat poser:

(1)

$$
\left(1+p t_{1}\right) \mu_{b}=\left(1+p t_{2}\right) p_{b}^{*}
$$

Cetto hypothise se justifie par lo fait que donx torroins de qualité diffé.

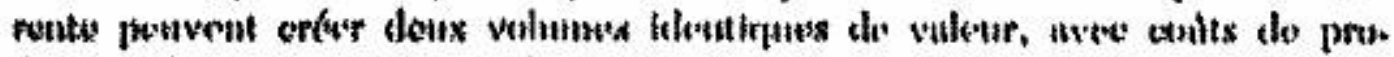

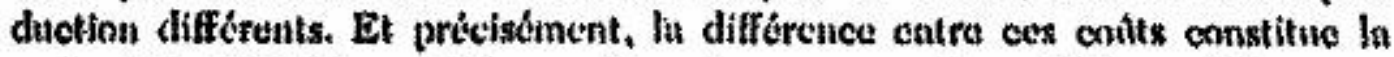
rente. L'égalité (1) peut ôtre posíc puisque sera la variable qui l'assurera ex poxt. lionulité (1) deviont:

$$
\begin{aligned}
& p_{b}^{t}+p_{b}^{t_{0}} \cdot p t_{1}=p_{b}^{2} \cdot p t_{2}+p_{b}^{2} \\
& \mu_{b}-\mu_{b}=\mu_{b} \cdot \mu_{2}-\mu_{b} \cdot \rho I_{1} \\
& \text { d'oi } p=\begin{array}{c}
p_{b}-p_{b}^{2} \\
p_{b}^{2} t_{2}-p_{0}^{1} t_{1}
\end{array}
\end{aligned}
$$

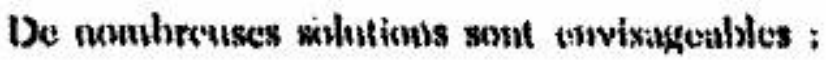

$$
\Lambda-S I p_{b}^{\prime}>p_{b}^{2} \Rightarrow p_{b}^{2} t_{2}>p_{b}^{s} t_{1}
$$

$$
\begin{aligned}
& \frac{m_{0}^{*}}{m_{0}}>\frac{t_{1}}{t_{3}} \\
& \frac{m_{0}^{*}}{m_{b}^{2}}<1
\end{aligned} \mid \Rightarrow \frac{t_{1}}{t_{2}}<1 \Rightarrow t_{1}<t_{2}
$$

$f_{2}$ cat la terre marginate

2) Divisons par $t_{1}$ puis patr $t_{2}$ l'inegulits $p_{6}^{2} t_{2}>\mu_{6} t_{1}$

a) $\nu_{b}^{2} \cdot \cdot \frac{t_{3}}{t_{1}}>\mu_{b}^{1}$

Comme par hypothèse $p_{0}^{1}>p_{b}^{2}{ }^{t_{2}}{ }_{t_{1}}^{t_{1}}$ the pout qu'être supéricur à lunité.

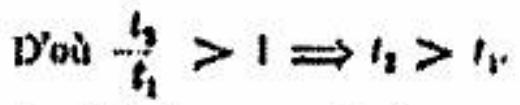

I. est la terro marginale. 


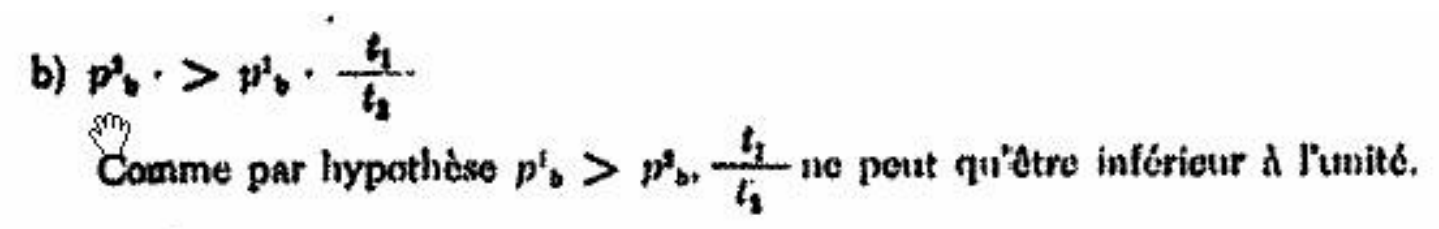

D'ou $\frac{t_{1}}{t_{2}}<1 \Rightarrow t_{2}>t_{2}$

$t_{2}$ est la terre marginale

B - Si $p_{b}^{1}<p_{b}^{2} \Rightarrow p_{b}^{*} \cdot l_{2}<p_{b}^{1} \cdot l_{1}$

1)

$$
\left.\begin{array}{l}
\frac{p_{b}}{p_{b}}<\frac{t_{1}}{t_{2}} \\
\frac{p_{b}^{2}}{p_{b}^{2}}>1
\end{array}\right\} \Rightarrow \frac{t_{1}}{t_{2}}>1 \Rightarrow t_{1}>t_{2},
$$

$t_{1}$ cat la terre marginale

2) Divisons par $t_{1}$, puis par $t_{2}$ M'inćgalité $p_{6}^{2} \cdot t_{2}<p_{6}^{3} \cdot t_{1}$

a) $p_{b}^{2} \cdot \frac{t_{3}}{t_{1}}<p^{t}$

$\frac{t_{2}}{t_{1}}$ ne peut otro qu'inférionr a liunite. D'oì $\frac{t_{2}}{t_{1}}<1 \Rightarrow t_{1}>t_{2}$ $t_{1}$ est la terre marginalo

b) $p_{0}^{2}<p_{0}^{2} . t_{3}$

$\frac{t_{1}}{t_{2}}$ ne peut être que supéricur \& l'umité. D'oì $\frac{t_{1}}{t_{1}}>\mathrm{j} \Rightarrow t_{1}>t_{2}$ 1. ent la terre marginale

\section{BIBLIOGRAPHIE}

(1980) ABRAHAM-FROIS G., BERREBI E., Rentes, rareté et surprofits. Paris, Economica (1983) ADDI L., «Rareté, rente et plus-value» in Temps modernes, mars (1979) Cahiers d'économie politique 5. PUF. Cf. les articles de G. Abraham-Frois et E. Berrebi, J. Cartelier, A. Lipietz, J.E. Mestre et D. Diatkine (1977) MONTANI G, «La notion ricardienne de la rente» in G. FACCARELLO et P. de LAVERGNE. Une nouvelle approche en économie politique?, Paris. Economica (1978) RICARDO D., Des principes de l'économie politique et de l'impôt, Paris, CarnierFlammarion.

(1972) SCHEFOLD B., «Le système de Sraffa et la production jointe: deux exemples d'application » in Ricardiens, keynésiens et marxistes, actes du colloque de Nice. Septembre (1970) SRAFFA P., Production des marchandises par les marchandises. Paris. Dunod (1977) VIDONNE P., "Une présentation critique de la rente ricardienne», Revue économique 2 , mars (1977) VIDONNE P., "La rente et l'étalon avec ressource rare et homogène », Revue économique 5, septembre 
[5] Dans l'agriculture, où la rigidité d'un facteur de production, en l'occurrence la terre fertile, entraine assez vite la décroissance des rendements, la concurrence nivelle les prix en référence du prix le plus élevé. Dans l'industrie, où les firmes peuvent accroître leurs capacités de production en bénéficiant de la loi des rendements croissants jusqu'à une limite maximale que fixe l'élasticité de la demande, la concurrence nivelle les coûts de production en éliminant les firmes les moins performantes. A moins d'une situation de monopole, dans l'industrie, la concurrence élimine les rentes, ce qui accroît les capacités potentielles de la demande

[6] Pour que la comparaison soit possible, nous raisonnons dans le cas de terrains qui produisent le même produit agricole. En cas de produits différents, l'ordre de fertilité peut ne pas correspondre à l'ordre de rentabilité. C'est évident.

[7] D. Ricardo (1978).

[8] Les ponctions généralisées sur la demande effective ne risquent-elles pas d'éteindre le processus de création de la valeur ? Non, parce que celui-ci arrive à contrarier la logique de la rente et imposer l'élargissement de l'accumulation. La prééminence de la rente sur le produit net, donc si la demande effective, est la caractéristique de l'économie sous-développée et non de l'économie développée reposant sur une dynamique de la croissance qui, précisément, contrarie les ponctions opérées par les rentes diverses

[9] Sraffa écrit: «Il ne faut pas oublier que l'absence de branches non fondamentales du système-étalon n'empêche pas ce dernier d'être équivalent dans ses effets au système originel à partir du moment où, comme nous l'avons vu au paragraphe 6 , leur présence ou leur absence ne fait pas de différence pour la détermination des prix et du taux de profit. » ((1970), p.33).

[10] En général, la mise en culture d'un nouveau terrain ne s'accompagne pas d'une augmentation de prix, car ce dernier est au niveau maximum que peut accepter la demande. La mise en culture du nouveau terrain peut ne rien rapporter, en absolu, pour le premier terrain : elle ne rapporte rien, en absolu, si les prix sont inchangés car elle augmente la rente relativement. En cas de hausse de prix, elle augmente la rente en absolu

[11]Cependant, au-delà de la rente foncière, la rareté ne se manifeste pas uniquement pour les terres agricoles. Elle concerne tous les produits susceptibles d'intéresser la demande effective. Aussi dans ce cas, M1 serait l'indicateur du niveau d'imperfection de la concurrence puisqu, en cas de concurrence pure et parfaite, $M 1$ devrait être nul avec $M_{a}=0, M_{b}=0$, $\mathrm{M}_{\mathrm{c}}=0 \ldots$ simultanément. 
Contrairement à ce que pense A. Lipietz («Le mystère de la rente absolue » in Cahier d'économie politique 5, 1979), nous ne considérons pas la rareté comme une catégorie exclusivement marginaliste. Cf. Addi (1983)

[12] P. Vidonne (1977)

[13] P. Vidonne (1977)

[14] B. Schefold (1972)

[15] C'est sur ces prémisses de G. Montani et de B. Schefold que G. Abraham-Frois et E. Berribi (1980) construisent leur axiomatique consacrée par le théorème selon lequel « la rente $\mathrm{p}(\mathrm{r})$ est positive si et seulement si la méthode qui a la productivité économique en blé la plus grande a le prix de production le plus élevé ».

[16] Cet article résume une partie d'un travail personnel de recherche intitulé « Rente et plusvalue . Eléments critiques ", multigraphié, 250p., inédit. 